\title{
Plasma Parameters Effects on Dust Acoustic Solitary Waves in Dusty Plasmas of Four Components
}

\author{
Abeer A. Mahmoud, Essam M. Abulwafa $\mathbb{D}^{D}$, Abd-alrahman F. Al-Araby, \\ and Atalla M. Elhanbaly \\ Theoretical Physics Research Group, Physics Department, Faculty of Science, Mansoura University, Mansoura 35516, Egypt \\ Correspondence should be addressed to Essam M. Abulwafa; abulwafa@mans.edu.eg
}

Received 26 July 2018; Accepted 11 September 2018; Published 27 September 2018

Academic Editor: P. Areias

Copyright (c) 2018 Abeer A. Mahmoud et al. This is an open access article distributed under the Creative Commons Attribution License, which permits unrestricted use, distribution, and reproduction in any medium, provided the original work is properly cited.

\begin{abstract}
The presence and propagation of dust-acoustic solitary waves in dusty plasma contains four components such as negative and positive dust species beside ions and electrons are studied. Both the ions and electrons distributions are represented applying nonextensive formula. Employing the reductive perturbation method, an evolution equation is derived to describe the smallamplitude dust-acoustic solitons in the considered plasma system. The used reductive perturbation stretches lead to the nonlinear $\mathrm{KdV}$ and modified $\mathrm{KdV}$ equations with nonlinear and dispersion coefficients that depend on the parameters of the plasma. This study represents that the presence of compressive or/and rarefactive solitary waves depends mainly on the value of the firstorder nonlinear coefficient. The structure of envelope wave is undefined for first-order nonlinear coefficient tends to vanish. The coexistence of the two types of solitary waves appears by increasing the strength of nonlinearity to the second order using the modified KdV equation.
\end{abstract}

\section{Introduction}

The dust acoustic solitary waves (DASWs) propagation (known as weak frequency-dusty plasma density vibrations) attracted the researchers since many years due to its eventual functions in different implementations. These implementations are such as lower ionosphere of the Earth, lower and upper mesosphere, asteroid zones, space environments, comet tails, interplanetary spaces and interstellar media [1-4]. In addition, it is instituting in different industrial process and laboratory devices like solar cells, semiconductor chips, plasma devices, fusion devices and so on [5-7]. Many researchers found that the spectra of the plasma waves are modified because of the existence of dust species. Ion waves in dusty plasma are studied by Angelis et al. [1] and they founded the equation, which described the perturbations due to the dust particles inhomogeneity distributions. Their results are employed to analyze the data for Halley's Comet collected from Vega and Giotto space probes. Rao et al. [8] showed theoretically that a new kind of dust acoustic waves (DAWs) propagates through dusty plasma with linear and nonlinear modes known as supersonic solitons. The DAW has been studied experimentally in laboratory under microgravity conditions by Piel and coworkers [7, 9]. Cassini spacecraft had taken high-resolution images for the Saturn's rings. These images investigated for proof of Mach cons DAW, which could supply information on the physical properties of the ring particles [10].

Since the existence of the dust charged particles was not neglected, because not only they modify the spectra of the plasma but also they introduce new eigenmodes such as dust ion acoustic waves (DIAWs), DAWs, dust lattice waves (DLWs), dust cyclotron acoustic, and dust drift mode, etc. [11-14]. Mamun et al. [15] studied twocomponent dusty plasma with Boltzmann distributed ions. They found that only negative solitary wave is admitted where the Kortweg-de Vries (KdV) equation was derived. In addition, they studied the same dusty plasma with vortexlike or nonthermal distribution for ions [16]. They concluded that the small amplitude dynamics of this system were described via the modified Kortweg-de Vries (mKdV) equation. Mamun [17] studied the ion temperature effect 
on the electrostatic solitons in nonthermal plasmas, which contain nonthermally distributed electrons and warm adiabatic ions using Sagdeev potential technique. It was noticed that ion temperature affects the Mach number for which the solitons exist and the nonthermal parameter. In addition, both the width and the amplitude of these solitary waves are affected.

There are broad mathematical ways to deal with waves in dusty plasma, but there are two major methods used. Studying the arbitrary amplitude of waves in plasmas using the Sagdeev pseudo-potential (SPP) approach is the most common way [18]. While for the small amplitude electrostatic waves, a reductive perturbation theory (RPT) can be employed to extract the evolution equation [19]. Mamun [20] applied the RPT to study the properties of the DAWs for unmagnetized three-component dusty plasma system composed of dusty grain carries negative charge beside Boltzmann distributed electrons and ions. Bedi et al. [21] hired the reductive perturbation method to deduce the $\mathrm{KdV}$ equation for four-component magnetized dusty plasma consisting of non-thermal electrons; Maxwellian distributed ions and two dust grains with negative and positive charges. Tasnim et al. [22] studied the propagation of electrostatic potential through adiabatic dusty plasma consisting of four components such as adiabatic electrons, adiabatic ions, adiabatic dust species of positive charge, and negatively charged warm dust grains. Akhter et al. [23] utilized the RPT to drive the KdV equation for magnetized dusty plasma composed of four components such ions and electrons with Maxwellian distributions alongside negatively and positively charged dust grains. They studied the effects of different dust plasma parameters on the dust acoustic solitary structures. Sebastian et al. [24] investigated the DA solitary waves in five-component dusty plasma system contain hot electrons and ions, kappa distributed photoelectrons, and two dust species of positive and negative charges.

Due to Boltzmann-Gibbs entropy generalization in the nonequilibrium states with the nonextensive entropy suggested by Tsallis [25], many researchers have paid more attention to employ the nonextensive distribution for the number density of particles in plasma [26-28].

Through this paper, a dusty plasma system composed of four components such as electrons and ions described by $q$-nonextensive distributions beside two species of dust having positive and negative charges is studied. The reductive perturbation method is applied to investigate the small amplitude electrostatic structures through this plasma system. The effects of some different plasma parameters (traveling wave velocity, nonextensive parameter, and dust temperature ratios) on the electrostatic solitary wave structure (shape, width, and height) are inspected.

The rest of the paper is ordered as follows: fluid equations that describe the considered plasma system are represented in Section 2. Through Sections 3 and 4, RPT is appointed to deduce the evolution equations that describe electrostatic waves propagate in the system. Section 5 is devoted for conclusion on the obtained results.

\section{Fluid Equations for the Plasma System}

A system of dusty plasma composed of ions and electrons with nonextensive distributions along with negative and positive dust sorts is treated in the following. The onedimensional fluid equations that depict this kind of plasma are represented as $[20,29-31]$ :

$$
\begin{aligned}
& \frac{\partial}{\partial t} N_{j}(x, t)+\frac{\partial}{\partial x}\left[N_{j}(x, t) U_{j}(x, t)\right]=0 \\
& \beta_{j}\left[\frac{\partial}{\partial t} U_{j}(x, t)+U_{j}(x, t) \frac{\partial}{\partial x} U_{j}(x, t)\right]+\frac{\partial}{\partial x} \phi(x, t) \\
& \quad+\frac{\sigma_{j}}{N_{j}(x, t)} \frac{\partial}{\partial x} P_{j}(x, t)=0 \\
& \frac{\partial}{\partial t} P_{j}(x, t)+U_{j}(x, t) \frac{\partial}{\partial x} P_{j}(x, t) \\
& \quad+3 P_{j}(x, t) \frac{\partial}{\partial x} U_{j}(x, t)=0 .
\end{aligned}
$$

where $j=p$ for the positive dusty fluid and $j=n$ for the negative one. $N_{j}(x, t)$ refers to the dust fluid density number normalized by its equilibrium value $N_{j 0} ; U_{j}(x, t)$ is the dust particle velocity normalized by the dust-acoustic velocity $C_{s}=\sqrt{Z_{n} k_{B} T_{i} / m_{n}} \cdot \beta_{p}=Z_{n} m_{p} /\left(Z_{p} m_{n}\right)=\mu$ and $\beta_{n}=1 . Z_{j}$ is the electronic charges number on the dust grain surface, $m_{j}$ is the dust particle mass, $T_{i}$ is the ions temperature, and $k_{B}$ is the Boltzmann constant. $P_{j}(x, t)$ is the dust particles pressure normalized by $N_{j 0} k_{B} T_{j}$ and the dust temperatures ratio is $\sigma_{j}=T_{j} /\left(Z_{j} T_{i}\right)$, where $T_{j}$ is the jth dust fluid temperature. $\phi(x, t)$ is the electrostatic potential normalized by $k_{B} T_{i} / e . x$ and $t$ are the space and time variables, which are normalized with the positive dust Debye length $\lambda_{D p}=\sqrt{k_{B} T_{i} /\left(4 \pi N_{n 0} e^{2} Z_{n}\right)}$ and inverse frequency $\omega_{p}^{-1}=\sqrt{m_{n} /\left(4 \pi N_{n 0} e^{2} Z_{n}\right)}$, respectively.

Equations ((1a), (1b), and (1c)) consist a set of six coupled nonlinear partial differential equations in seven unknown functions. To make the used plasma system equations ((1a), (1b), and (1c)) self-consistent, Poisson's equation is proceeded as

$$
\begin{aligned}
\frac{\partial^{2}}{\partial x^{2}} \phi & (x, t)-\mu_{e} N_{e}(x, t)-N_{n}(x, t)+\mu_{i} N_{i}(x, t) \\
& +\mu_{p} N_{p}(x, t)=0
\end{aligned}
$$

where $\mu_{e}=N_{e 0} /\left(N_{n 0} Z_{n}\right), \mu_{i}=N_{i 0} /\left(N_{n 0} Z_{n}\right)$, and $\mu_{p}=$ $N_{p 0} Z_{p} /\left(N_{n 0} Z_{n}\right)$ satisfy the neutrality condition $\mu_{p}+\mu_{i}-$ $\mu_{e}=1$, where $N_{i 0}$ and $N_{e 0}$ are the equilibrium density values of ions and electrons, respectively. $N_{i}(x, t)$ and $N_{e}(x, t)$ are the ions and electrons fluid densities, which are taken by $q-$ nonextensive distribution forms as

$$
N_{l}(x, t)=\left[1+\sigma_{l}\left(q_{l}-1\right) \phi(x, t)\right]^{\left(q_{l}+1\right) /\left[2\left(q_{l}-1\right)\right]},
$$

where the subscript $l$ refers to $i$ for ions and $e$ for electrons, $\sigma_{i}=-1, \sigma_{e}=T_{i} / T_{e}$ and $T_{e}$ is the electrons temperature. 
Equations (1a)-(3) are complicated coupled nonlinear partial differential equations, so the reductive-perturbation method will be employed to discuss the acoustic waves of small-amplitude through our system of plasma.

\section{Small-Amplitude Waves and KdV Equation}

To examine the properties of dust acoustic solitons of smallamplitude, the reductive perturbation theory $[22,23]$ is utilized with the stretching variables:

$$
\begin{aligned}
\tau & =\varepsilon^{3 / 2} t, \\
\xi & =\varepsilon^{1 / 2}(x-\lambda t),
\end{aligned}
$$

where $\varepsilon$ represents the amplitude of the perturbation and $\lambda$ is the phase speed of the wave. All the plasma parameters $\Psi(x, t)$ $=\left[N_{j}(x, t), U_{j}(x, t), P_{j}(x, t), \phi(x, t)\right]$ appearing in (1a)-(3) are expanding as a power series in $\varepsilon$ about their equilibrium values as

$$
\Psi(x, t)=\sum_{n=0}^{\infty} \varepsilon^{n} \psi_{n}(x, t),
$$

with the conditions $N_{p}=N_{n}=P_{p}=P_{n}=1$ and $U_{p}=U_{n}=$ $\phi=0$ as $|\xi| \longrightarrow \infty$ and with realization of the neutrality condition. Substituting (4) and (5) into (1a)-(3) and equating the coefficients of similar powers of $\varepsilon$, one has the following.

The coefficients of first order of $\varepsilon$ give relations between the first perturbed orders of all physical quantities. The first perturbed order of Poisson's equation gives the following compatibility condition (linear dispersion relation (LDR)) as

$$
\begin{aligned}
L D R 1 & =\frac{\mu_{p}}{\left(\lambda^{2} \mu-3 \sigma_{p}\right)}+\frac{1}{\left(\lambda^{2}-3 \sigma_{n}\right)}+\alpha_{1}=0, \\
\alpha_{1} & =-\frac{\left[\mu_{i}\left(q_{i}+1\right)+\mu_{e} \sigma_{e}\left(q_{e}+1\right)\right]}{2} .
\end{aligned}
$$

This linear dispersion relation should be solved to derive the acoustic phase speed $\lambda$ in terms of the plasma parameters as

$$
\lambda_{ \pm}=\left[\frac{\left\{3 \alpha_{1}\left(\sigma_{p}+\mu \sigma_{n}\right)-\left(\mu+\mu_{p}\right) \pm \sqrt{\left[3 \alpha_{1}\left(\sigma_{p}-\mu \sigma_{n}\right)-\left(\mu+\mu_{p}\right)\right]^{2}+12 \alpha_{1} \mu\left(\sigma_{p}-\mu \sigma_{n}\right)}\right\}}{\left(2 \alpha_{1} \mu\right)}\right]^{1 / 2} .
$$

The positive sign refers to fast acoustic speed while the negative one represents slow acoustic speed $[32,33]$. In our numerical calculations, we considered the case of low acoustic speed whereas the fast acoustic speed is completely ignored. Also, it can be observed that the fast acoustic speed vanishes in the limiting case as $\sigma_{p}=\sigma_{n}=0$.

The next $\varepsilon$-order handles a set of differential equations, by eliminating the second-order of the perturbed quantities $\psi_{2}(\xi, \tau)$; the following Kortweg de-Vreis equation is obtained as

$$
\frac{\partial \phi(\xi, \tau)}{\partial \tau}+A \phi(\xi, \tau) \frac{\partial \phi(\xi, \tau)}{\partial \xi}+B \frac{\partial^{3} \phi(\xi, \tau)}{\partial \xi^{3}}=0
$$

where $\phi(\xi, \tau)=\phi_{1}(\xi, \tau)$ and $A$ and $B$ are the firstorder nonlinear coefficient and the dispersion coefficient, respectively, which are specified by the following formulae:

$$
\begin{aligned}
A & =\left[\frac{3 \mu_{p}\left(\lambda^{2} \mu+\sigma_{p}\right)}{\left(\lambda^{2} \mu-3 \sigma_{p}\right)^{2}}+\frac{2 \lambda}{\left(\lambda^{2}-3 \sigma_{n}\right)^{2}}+2 \alpha_{2}\right] B, \\
B & =\frac{1}{\left\{2 \lambda\left[\mu \mu_{p} /\left(\lambda^{2} \mu-3 \sigma_{p}\right)^{2}+1 /\left(\lambda^{2}-3 \sigma_{n}\right)^{2}\right]\right\}}, \\
\alpha_{2} & =-\frac{\left[\mu_{i}\left(q_{i}+1\right)\left(q_{i}-3\right)+\mu_{e} \sigma_{e}^{2}\left(q_{e}+1\right)\left(q_{e}-3\right)\right]}{8} .
\end{aligned}
$$

The stationary solitary wave solution of (7a) is obtained using the following traveling wave transformation:

$$
\begin{gathered}
\zeta=\xi-v \tau, \\
\Phi(\zeta)=\phi(\xi, \tau),
\end{gathered}
$$

where $v$ is the traveling wave velocity. The KdV equation (7a) becomes ordinary differential equation, which are known by the reduced $\mathrm{KdV}$ equation in the following form:

$$
-v \frac{d \Phi(\zeta)}{d \zeta}+A \Phi(\zeta) \frac{d \Phi(\zeta)}{d \zeta}+B \frac{d^{3} \Phi(\zeta)}{d \zeta^{3}}=0
$$

The well-known solution of (10) is represented by the following form:

$$
\Phi(\zeta)=\Phi_{m} \operatorname{sech}^{2}\left(\frac{\zeta}{\Delta}\right)
$$

where

$$
\begin{aligned}
\Phi_{m} & =\frac{3 v}{A}, \\
\Delta & =\sqrt{\frac{4 B}{v}} .
\end{aligned}
$$


Integrating (10) with boundary conditions $\Phi(\zeta) \rightarrow 0$, $d \Phi(\zeta) / d \zeta \longrightarrow 0$ and $d^{2} \Phi(\zeta) / d \zeta^{2} \longrightarrow 0$ as $\zeta \longrightarrow \pm \infty$, one gets

$$
\frac{1}{2}\left[\frac{d \Phi(\zeta)}{d \zeta}\right]^{2}+V_{s}(\Phi)=\mathrm{E}
$$

This is the form of a conservation law. The right-hand side $\mathrm{E}$ is the constant of integration, which is supposed as the total energy of a hypothetical body with unit mass, while the first term in the left-hand side is assumed as kinetic energy while the second term represents a potential energy that has the following form:

$$
V_{s}(\Phi)=-\left[\frac{v}{(2 B)}\right] \Phi(\zeta)^{2}+\left[\frac{A}{(6 B)}\right] \Phi(\zeta)^{3}
$$

This potential has the characteristics that its value and first derivative at $\Phi=0$ vanish while the second derivative is less than zero where both $v$ and $B$ have positive values. This means that the potential has a root and maximum value at the origin of motion. The other root of the potential is given as $\Phi=3 v / A=\Phi_{m}$, which is the amplitude of the solitary wave. The amplitude value is negative or positive depending mainly on the value of $A$ (the nonlinear coefficient), which depends on the plasma parameters. Therefore, plasma system provides two types of potential structure known as rarefactive (dark) solitons or compressive (bright). The dark (rarefactive) soliton exists if $A<0$ while the bright (compressive) soliton exists if $A>0$.

The change of the coefficient of nonlinear term $A$ with different plasma parameters (non-extensive parameter $q$, positive dust temperatures ratio $\sigma_{p}$, and negative dust temperatures ratio $\sigma_{n}$ ) shows that the existence of the two sorts of solitons (compressive and rarefactive) depends mainly on the values of these parameters. To investigate the influences of plasma parameters (nonextensive parameter $q$ and positive and negative dust temperatures ratios $\sigma_{p}$ and $\sigma_{n}$ ), the other plasma parameters are used as $\mu=2.0, \mu_{p}=0.7$, $\mu_{e}=0.2, \sigma_{e}=0.5[34,35]$. Figure $1(\mathrm{a})$ shows that the soliton converted from compressive to rarefactive at nonextensive parameter value depending on the positive dust temperature ratio as shown in Figure 1(a) and on the negative dust temperature ratio as appeared in Figure 1(b). We have discussed the impacts of these different plasma parameters on the shape structure of the envelope soliton by sketching in two dimensions both the small amplitude potential $V_{s}(\Phi)$ versus electrostatic potential $\Phi$ and the first perturbed electrostatic potential $\Phi(\zeta)$ versus travelling wave parameter $\zeta$ as represented in Figures 2-5. It is seen that from
Figure 2 one type of solitons only envelopes with varying the travelling wave velocity $v$, while the amplitude (width) of the wave increases (decreases) with increasing $v$ without effect on changing from soliton type to another. It is obvious from Figures 3, 4, and 5 that the compressive soliton amplitude and width increase with $q$ and $\sigma_{n}$ while they decrease with $\sigma_{p}$. On the other hand, the amplitude and width of rarefactive soliton decrease with both $q$ and $\sigma_{n}$ but increase with $\sigma_{p}$. Figures $3-5$ read that the soliton type transferred from compressive to rarefactive depending on the values of the plasma parameters $q, \sigma_{p}$ and $\sigma_{n}$ at which the nonlinear parameter $A$ tends to zero. Therefore, nearly at $A \approx 0$, this system cannot be described and a higher order nonlinearity evolution equation must be considered to describe the system in this region. This evolution equation will be described in the following section.

\section{Small Amplitude Waves and the Modified KdV Equation}

To study the solitary waves at the critical region, the following stretching coordinates are as

$$
\begin{aligned}
& \tau=\varepsilon^{3} t, \\
& \xi=\varepsilon(x-\lambda t) .
\end{aligned}
$$

Substituting (5) and (14) into (1a)-(3) and comparing the coefficients of same powers of $\varepsilon$, the coefficients of second order of $\varepsilon$ give relations between the first-order and the second-order perturbed physical quantities. The coefficients of the third order of $\varepsilon$ give a set of equations in terms of the first-, second-, and third-order perturbed quantities. These relations lead to another linear dispersion relation of the following form:

$$
\begin{aligned}
\operatorname{LDR} 2 & =\frac{3 \mu_{p}\left(\lambda^{2} \mu-\sigma_{p}\right)}{\left[2\left(\lambda^{2} \mu-3 \sigma_{p}\right)^{3}\right]}-\frac{3\left(\lambda^{2}-\sigma_{n}\right)}{\left[2\left(\lambda^{2}-3 \sigma_{n}\right)^{3}\right]}+\alpha_{2} \\
& =0
\end{aligned}
$$

Eliminating the third-order perturbations $\psi_{3}(\xi, \tau)$ from the fourth-order perturbation coefficients of $\varepsilon$, the following modified Kortweg de-Vreis equation is obtained in the following form:

$$
\frac{\partial \phi(\xi, \tau)}{\partial \tau}+D \phi(\xi, \tau)^{2} \frac{\partial \phi(\xi, \tau)}{\partial \xi}+B \frac{\partial^{3} \phi(\xi, \tau)}{\partial \xi^{3}}=0,
$$

where $D$ is the second-order nonlinear coefficient, which is given by the following formulae:

$$
\begin{aligned}
& D=\left\{\frac{3 \mu_{p}\left(5 \lambda^{4} \mu^{2}+30 \lambda^{2} \mu \sigma_{p}+9 \sigma_{p}^{2}\right)}{\left[2\left(\lambda^{2} \mu-3 \sigma_{p}\right)^{5}\right]}+\frac{3\left(5 \lambda^{4}+30 \lambda^{2} \sigma_{n}+9 \sigma_{n}^{2}\right)}{\left[2\left(\lambda^{2}-3 \sigma_{n}\right)^{5}\right]}+3 \alpha_{3}\right\} B, \\
& \alpha_{3}=-\frac{\left[\mu_{i}\left(q_{i}+1\right)\left(q_{i}-3\right)\left(3 q_{i}-5\right)+\mu_{e} \sigma_{e}^{3}\left(q_{e}+1\right)\left(q_{e}-3\right)\left(3 q_{e}-5\right)\right]}{48} .
\end{aligned}
$$




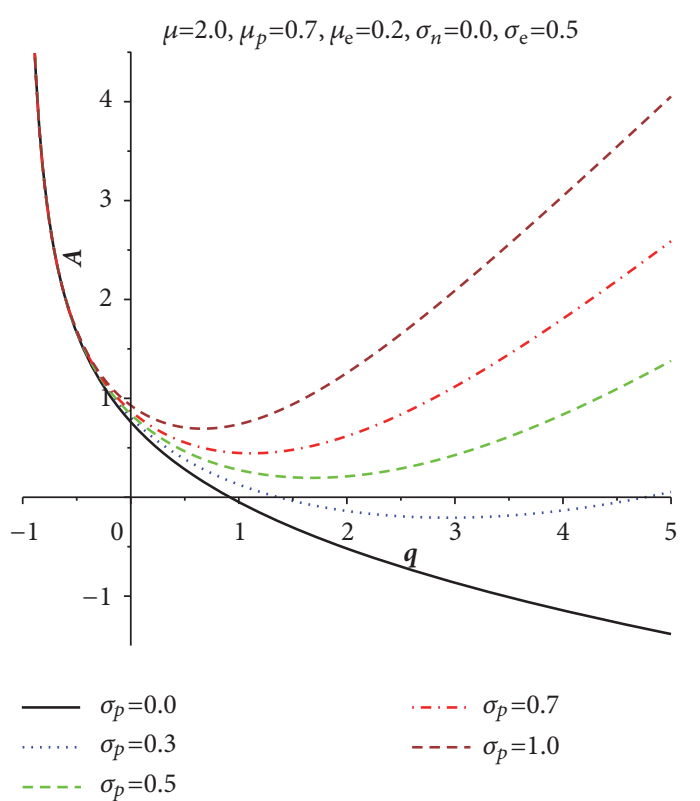

(a)

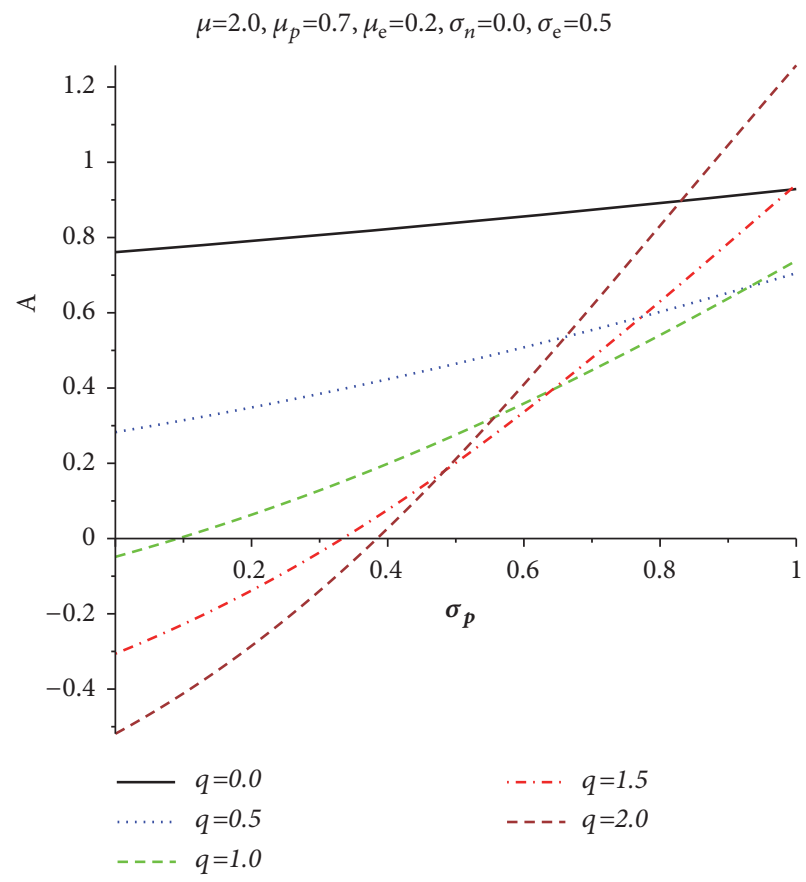

(c)

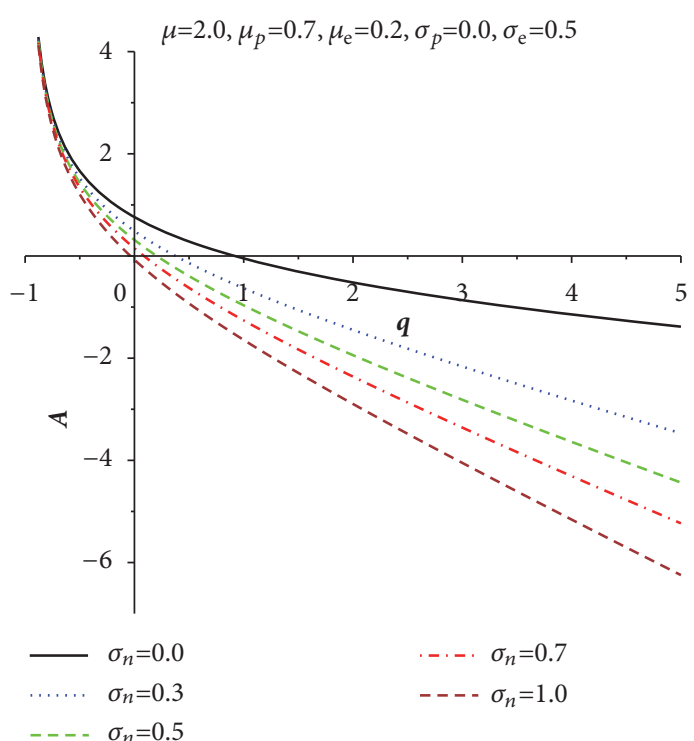

(b)

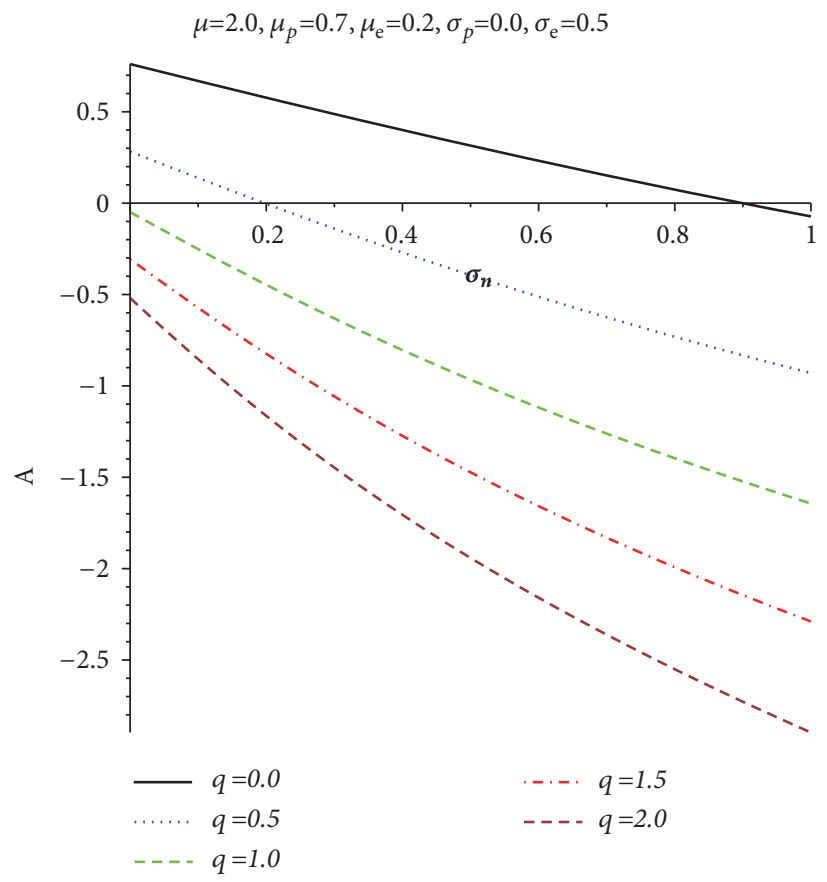

(d)

FIGURE 1

The stationary solitary wave solution of (16) can be deduced applying the traveling wave transformation (9). Therefore, the $\mathrm{mKdV}$ equation (16) is transformed into the following reduced $\mathrm{mKdV}$ equation:

$$
-v \frac{d \Phi(\zeta)}{d \zeta}+D \Phi(\zeta)^{2} \frac{d \Phi(\zeta)}{d \zeta}+B \frac{d^{3} \Phi(\zeta)}{d \zeta^{3}}=0,
$$

where $\Phi(\zeta)=\phi(\xi, \tau)$ with two solitary solutions as

$$
\Phi_{1}(\zeta)=\Phi_{m} \operatorname{sech}\left(\frac{\zeta}{\Delta}\right)
$$

$$
\Phi_{2}(\zeta)=-\Phi_{m} \operatorname{sech}\left(\frac{\zeta}{\Delta}\right)
$$

where $\Phi_{m}$ is the amplitude and $\Delta$ is the width of the wave envelop that are defined, respectively, as

$$
\begin{aligned}
\Phi_{m} & =\sqrt{\frac{6 M}{D}}, \\
\Delta & =\sqrt{\frac{4 B}{M}} .
\end{aligned}
$$




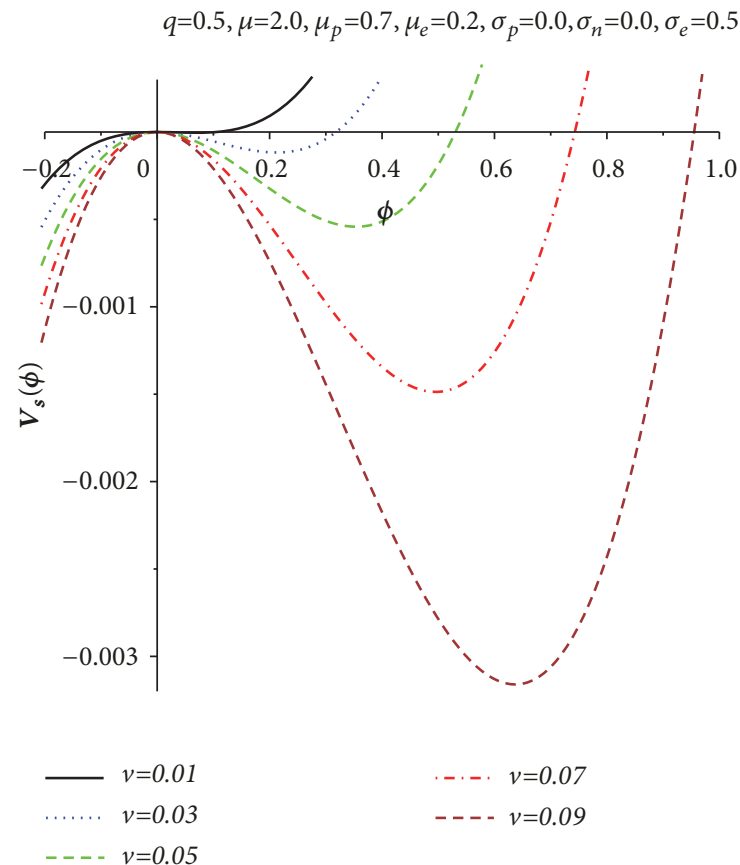

(a)

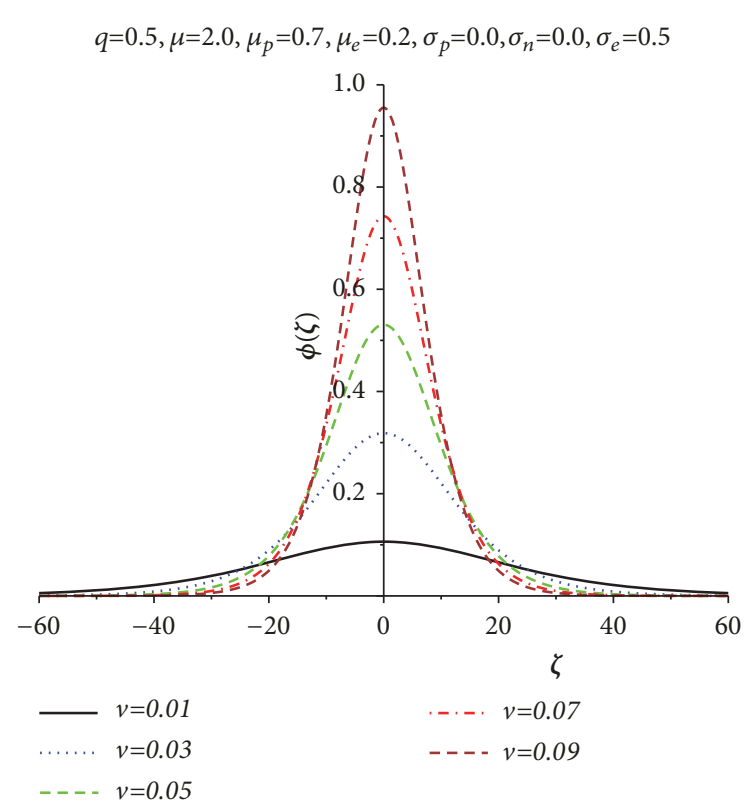

(b)

Figure 2

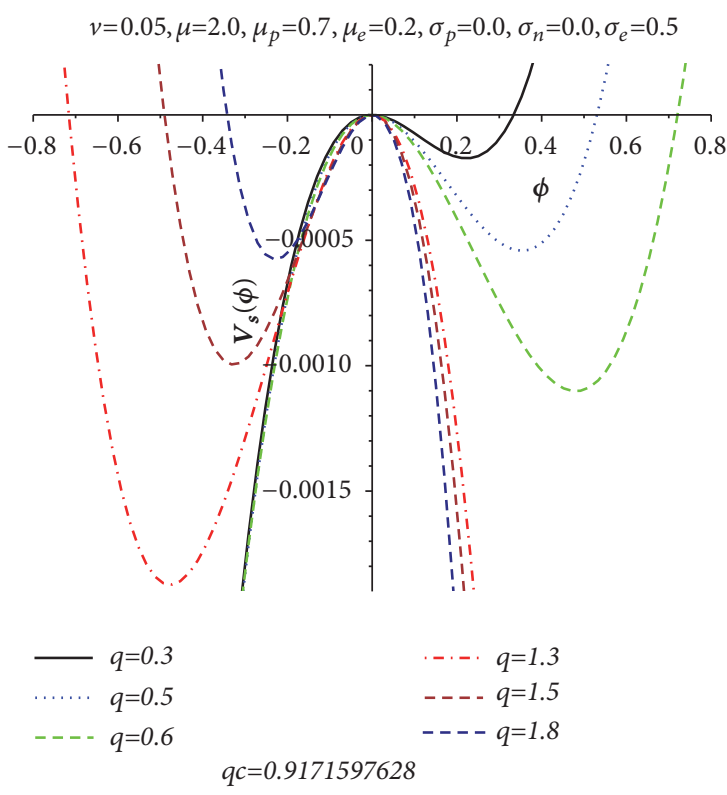

(a)

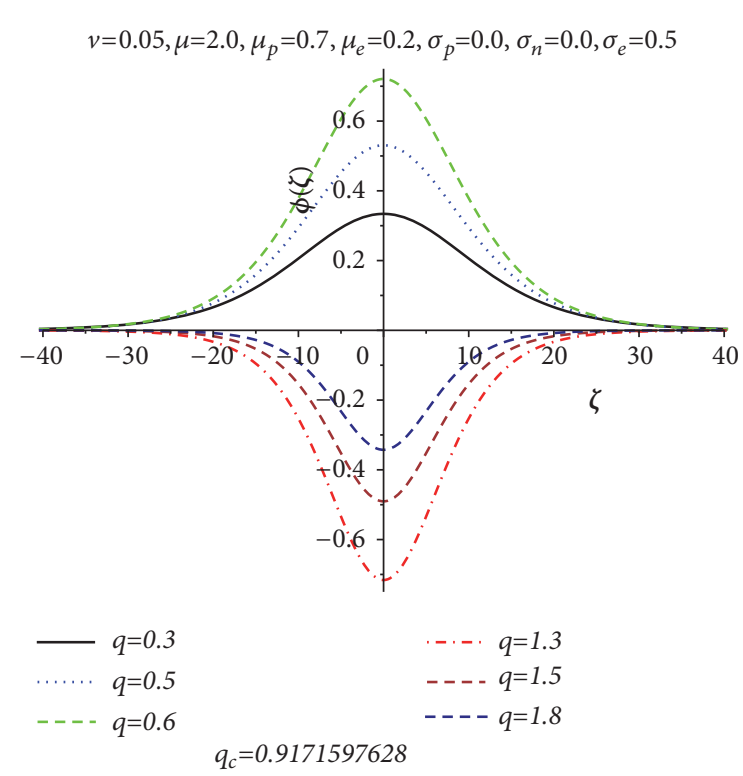

(b)

Figure 3

Here from the formulae of both amplitude and width of the envelop wave, we can say that, there is no envelop solitary wave for $D<0$ where $B>0$ from definition (8b).

Integrating (18) with boundary conditions $\Phi(\zeta) \longrightarrow 0$, $d \Phi(\zeta) / d \zeta \longrightarrow 0$ and $d^{2} \Phi(\zeta) / d \zeta^{2} \longrightarrow 0$ as $\zeta \longrightarrow \pm \infty$, one gets the small amplitude potential energy as

$$
V_{s}(\Phi)=-\left[\frac{v}{(2 B)}\right] \Phi(\zeta)^{2}+\left[\frac{D}{(12 B)}\right] \Phi(\zeta)^{4} .
$$

This potential has the characteristics that its value and first derivative at $\Phi=0$ vanish while the second derivative is negative. This means that there is a root and maximum value of $V_{s}(\Phi)$ at the origin. Both formulae ((19a), (19b)) and (20) appear the coexistence of the two types of solitons (compressive and rarefactive) in the region of vanishing the first-order nonlinear parameter $A$.

Here, we have treated the effects of non-extensive parameter $q$, positive dust temperature ratio $\sigma_{p}$ and negative dust 


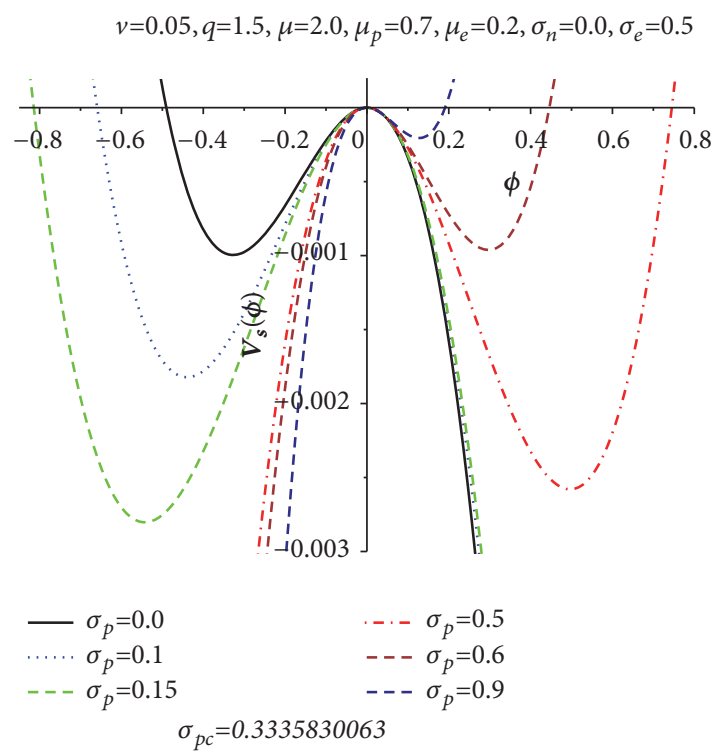

(a)

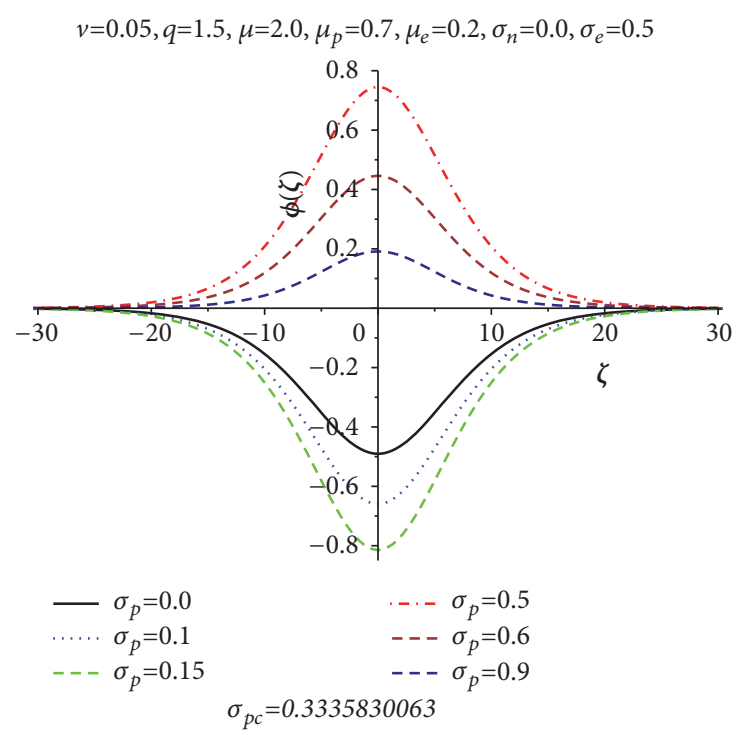

(b)

Figure 4

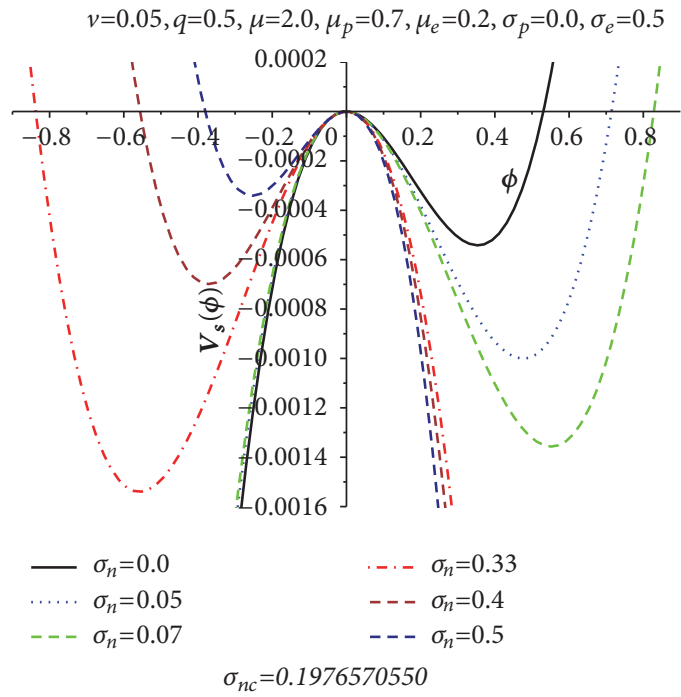

(a)

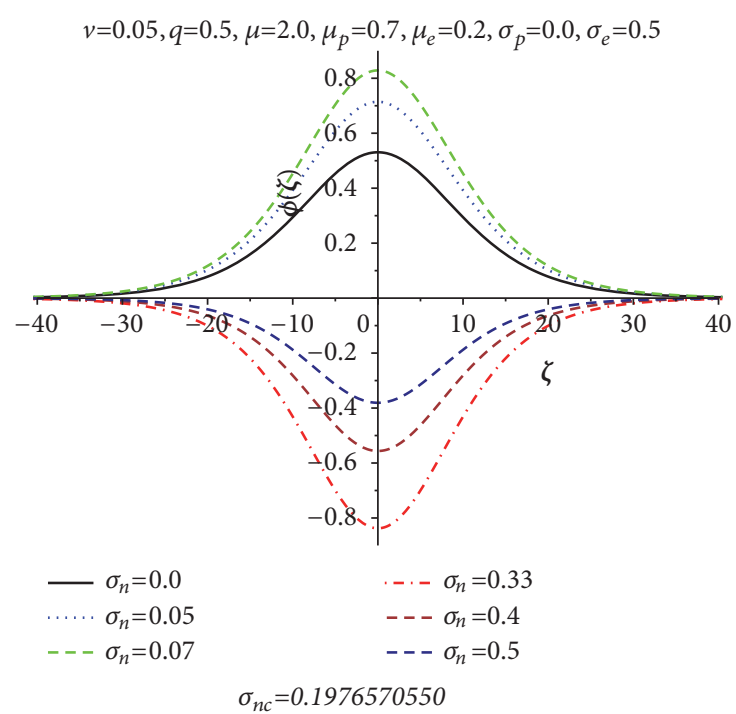

(b)

FIGURE 5

temperature ratio $\sigma_{n}$ with other real plasma parameters used as $\mu=2.0, \mu_{p}=0.7, \mu_{e}=0.2$ and $\sigma_{e}=0.5[34,35]$. As shown in Figure 6, the nonlinear coefficient $D$ increases with each of $q, \sigma_{p}$ and $\sigma_{n}$, but as shown from (19b) that $D$ must be greater than zero to envelope a soliton wave solution. The coexistence of both compressive and rarefactive solitons is indicated from the plotting of both small amplitude potential $V_{s}(\Phi)$ and the electrostatic potential $\Phi(\zeta)$ in Figures 7-10. It is evident that the amplitude (width) of the two types of solitons increases (decreases) with the increasing of the travelling wave velocity $v$ as in Figure 7. Figures 8-10 represent that both the width and amplitude of soliton envelope decrease with increasing of $q, \sigma_{p}$ and $\sigma_{n}$.

\section{Conclusion}

A plasma system consisting of four components such as negative and positive dust species beside ions and electrons is studied in this work. The hydrodynamics equations for the two dust species with pressure terms are employed to depict the dust particles motion, while the electrons and ions number densities are described in terms of nonextensive 


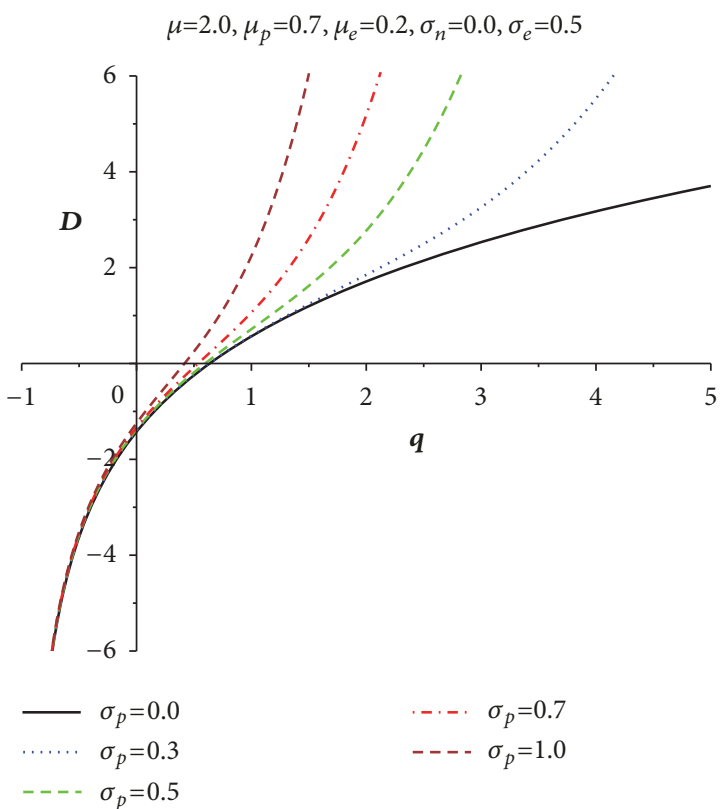

(a)

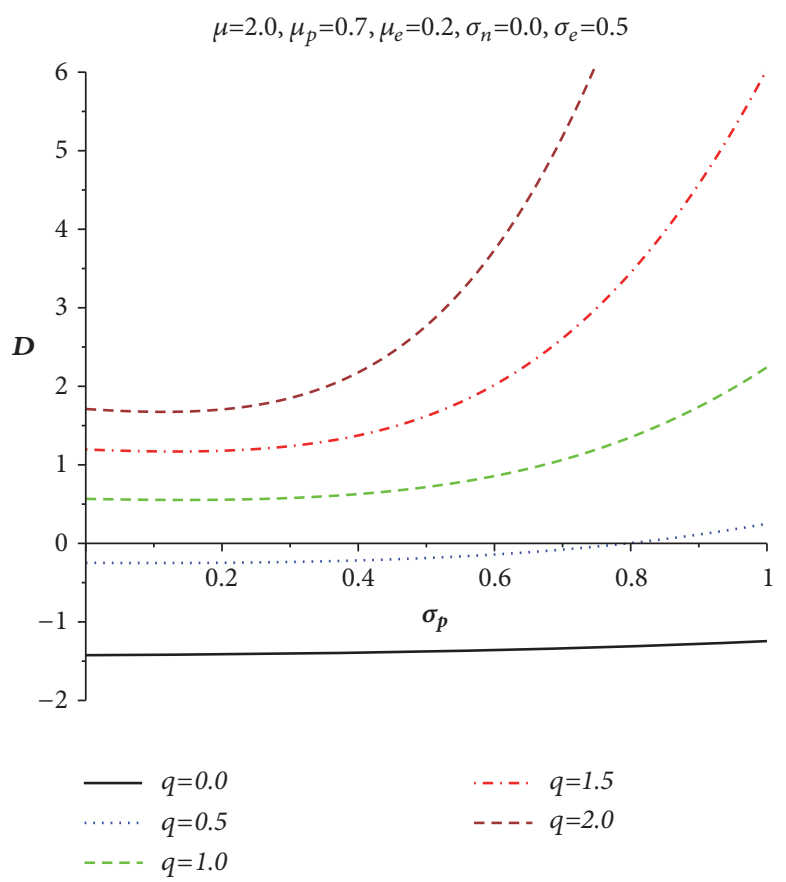

(c)

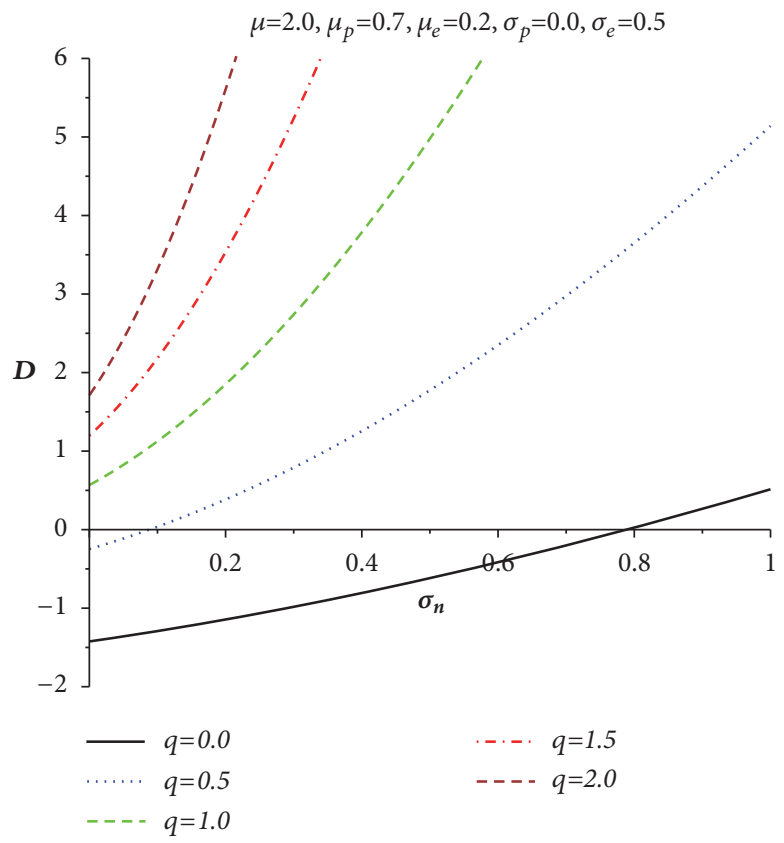

(b)

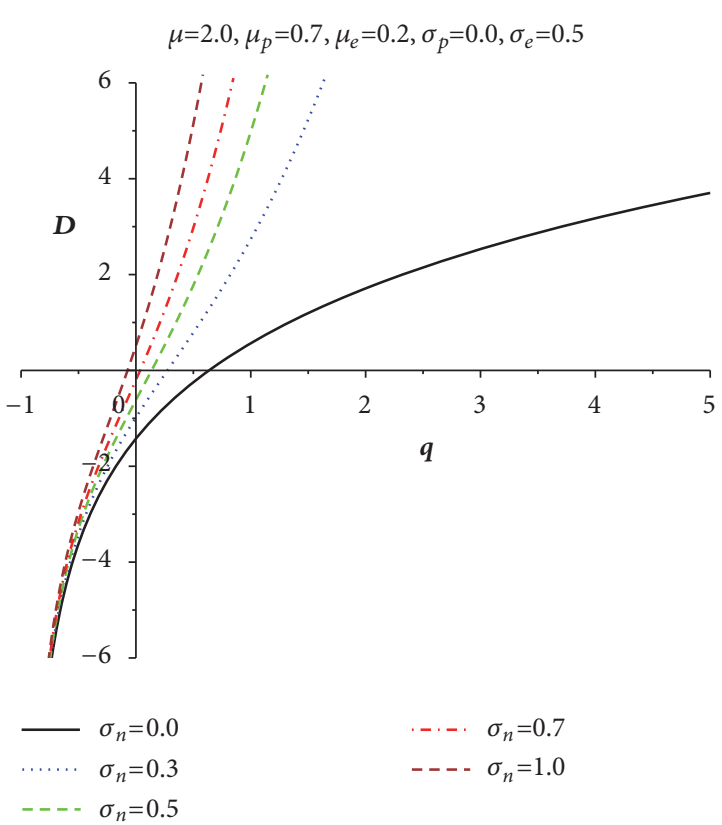

(d)

Figure 6

distributions. Poisson's equation is taking to make this system self-consistent.

The reductive perturbation method is utilized to deduce the KdV and $\mathrm{mKdV}$ equations that describe the small amplitude dust acoustic solitary waves propagated in our fourcomponent plasma system. These equations are integrated to give the equations of motion of a hypothetical particle of unit mass and virtual velocity $d \Phi / d \zeta$ under the effect of a virtual potential $V_{s}(\Phi)$ described by (13) and (20), where $\Phi(\zeta)$ is the electrostatic potential. In our numerical calculations, we considered the case of low acoustic speed whereas the fast acoustic speed completely vanishes in the limiting case as $\sigma_{p}=\sigma_{n}=0$.

The variation of the coefficients of nonlinear terms with different plasma parameters (the travelling wave velocity $v$, nonextensive parameter $q$, positive dust temperatures ratio $\sigma_{p}$, and negative dust temperatures ratio $\sigma_{n}$ ) shows the existence of two types of solitons (compressive and rarefactive). The soliton is compressive or rarefactive predicating mainly on the value of $A$ (first-order nonlinear coefficient), where 


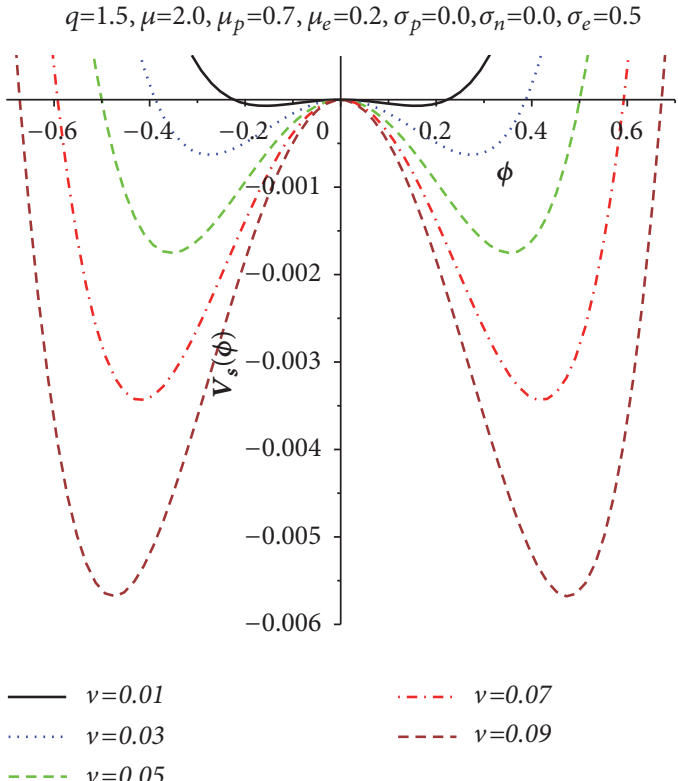

(a)
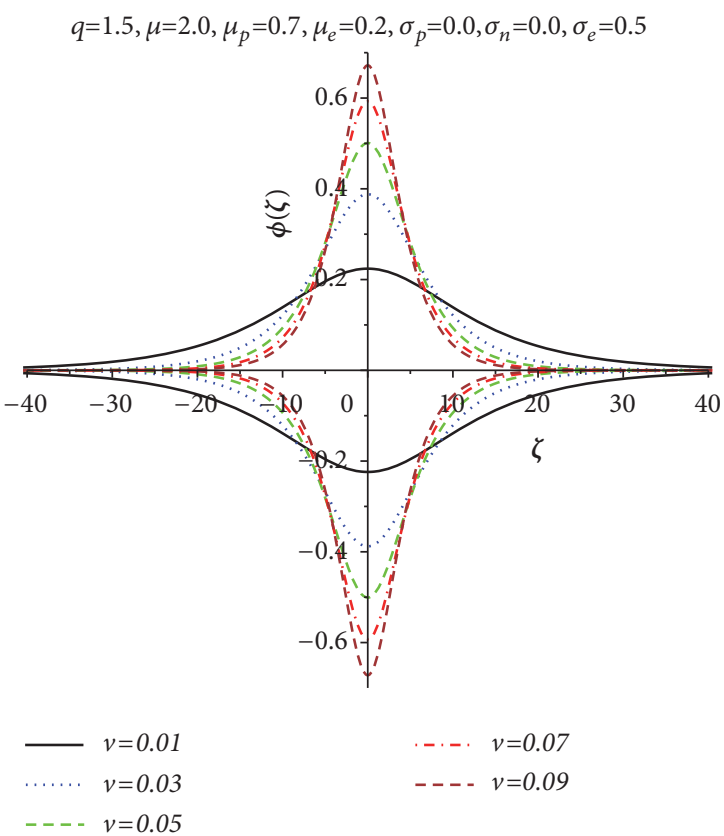

$\ldots-\ldots=0.05$

(b)

FIgURE 7

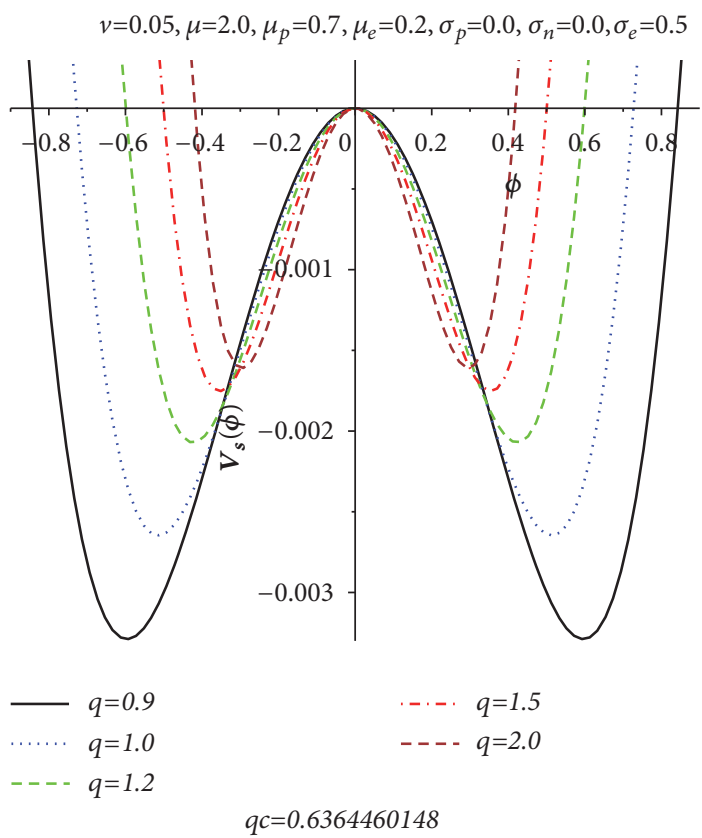

(a)

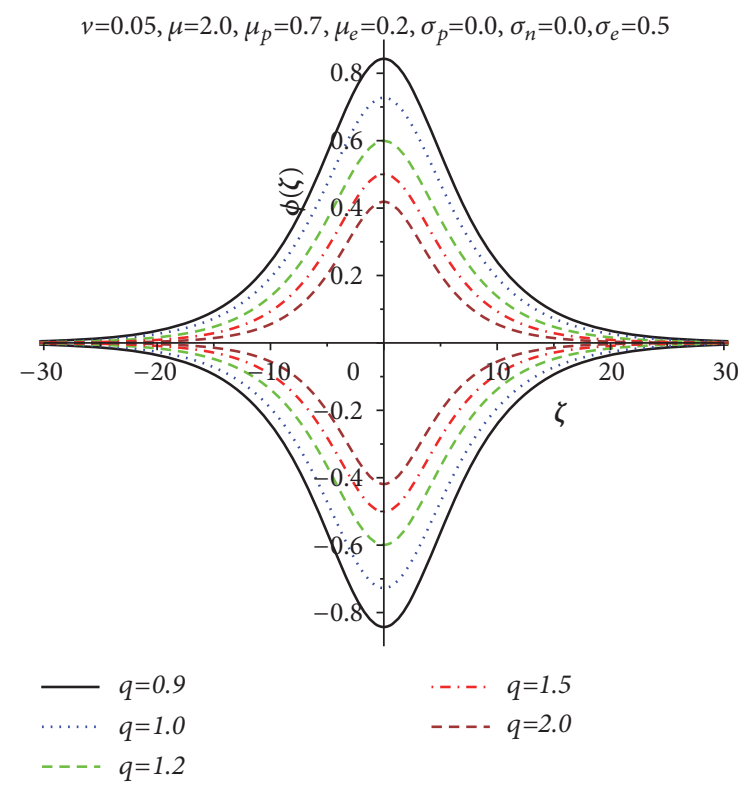

$q c=0.6364460148$

(b)

Figure 8

rarefactive soliton exists if $A<0$ while compressive soliton exists if $A>0$, but at $A \approx 0$, this system cannot be described. Therefore, the $\mathrm{mKdV}$ equation is considered to describe the system in this region. The solution of the $\mathrm{mKdV}$ equation represents the coexistence of both compressive and rarefactive solitons, which appears from the plotting of both the small amplitude potential $V_{s}(\Phi)$ and the electrostatic potential $\Phi(\zeta)$ for different values of the nonextensive 


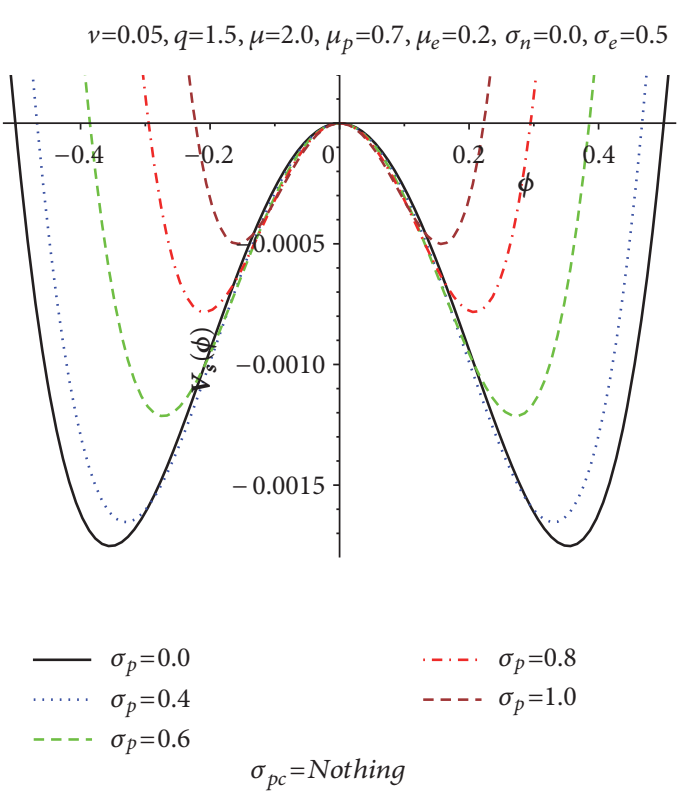

(a)

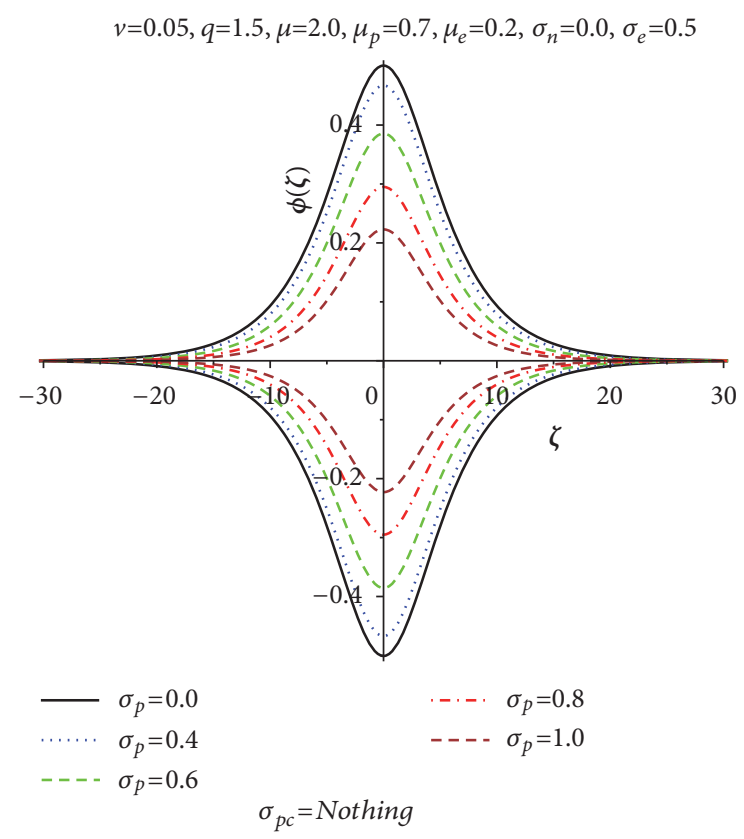

(b)

FIgURE 9

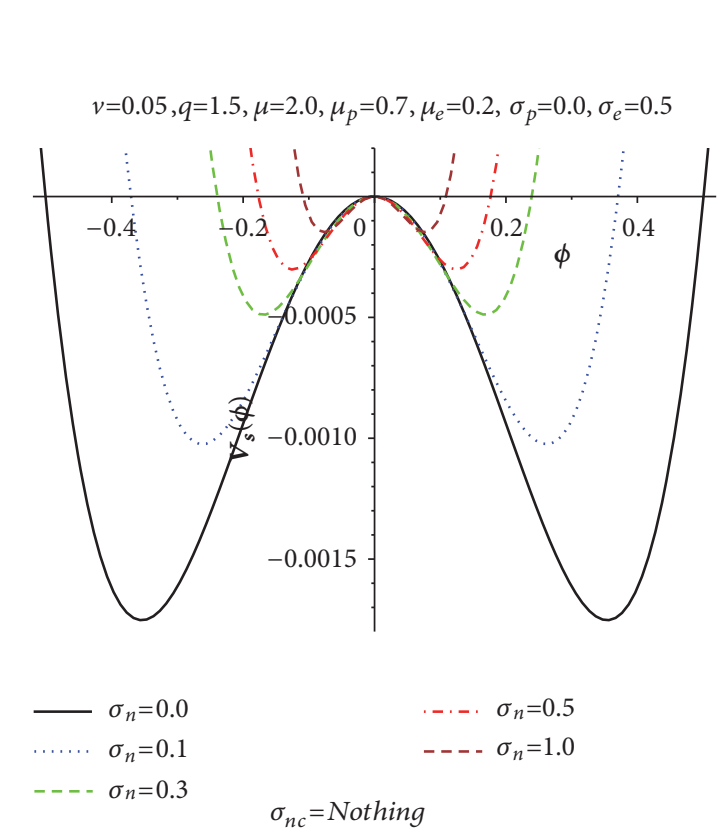

(a)

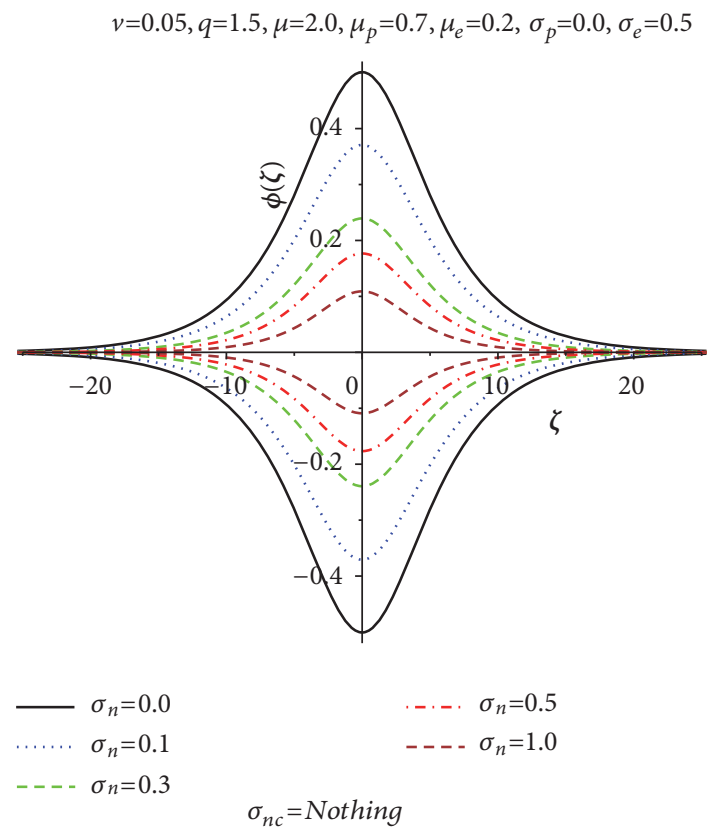

(b)

FIgURE 10

parameter $(q)$, and positive and negative dust temperature ratios $\left(\sigma_{p}\right.$ and $\left.\sigma_{n}\right)$.

\section{Data Availability}

The data used to support the findings of this study are included within the article.

\section{Conflicts of Interest}

The authors declare that they have no conflicts of interest.

\section{Acknowledgments}

The authors express their great acknowledgement to Prof. S. A. El-Wakil for his useful comments, continuous encouragement, and guidance. 


\section{References}

[1] U. de Angelis, V. Formisano, and M. Giordano, "Ion Plasma Waves in Dusty Plasmas: Halley's Comet," Journal of Plasma Physics, vol. 40, no. 3, pp. 399-406, 1988.

[2] P. K. Shukla, "Low-frequency modes in dusty plasmas," Physica Scripta, vol. 45, no. 5, pp. 504-507, 1992.

[3] F. Melandsø, T. K. Aslaksen, and O. Havnes, "A new damping effect for the dust-acoustic wave," Planetary and Space Science, vol. 41, no. 4, pp. 321-325, 1993.

[4] P. K. Kaw and A. Sen, "Low frequency modes in strongly coupled dusty plasmas," Physics of Plasmas, vol. 5, no. 10, pp. 3552-3559, 1998.

[5] A. Barkan, N. D’Angelo, and R. L. Merlino, "Experiments on ion-acoustic waves in dusty plasmas," Planetary and Space Science, vol. 44, no. 3, pp. 239-242, 1996.

[6] R. L. Merlino, A. Barkan, C. Thompson, and N. D’Angelo, "Laboratory studies of waves and instabilities in dusty plasmas," Physics of Plasmas, vol. 5, no. 5, pp. 1607-1614, 1998.

[7] T. Reichstein, I. Pilch, and A. Piel, "Toroidal dust motion in magnetized plasmas," Physics of Plasmas, vol. 17, Article ID 093701, 2010.

[8] N. N. Rao, P. K. Shukla, and M. Y. Yu, "Dust-acoustic waves in dusty plasmas," Planetary \& Space Science, vol. 38, no. 4, pp. 543-546, 1990.

[9] K. O. Menzel, O. Arp, and A. Piel, "Spatial frequency clustering in nonlinear dust-density waves," Physical Review Letters, vol. 104, no. 23, Article ID 235002, 2010.

[10] O. Havnes, T. Aslaksen, T. W. Hartquist et al., "Probing the properties of planetary ring dust by the observation of Mach cones," Journal of Geophysical Research: Space Physics, vol. 100, no. A2, pp. 1731-1734, 1995.

[11] F. Melandso, "Lattice waves in dust plasma crystals," Physics of Plasmas, vol. 3, no. 11, pp. 3890-3901, 1996.

[12] A. Homann, A. Melzer, S. Peters, R. Madani, and A. Piel, "Laserexcited dust lattice waves in plasma crystals," Physics Letters A, vol. 242, no. 3, pp. 173-180, 1998.

[13] P. K. Shukla and H. U. Rahman, "Instability of electrostatic dustcyclotron waves and associated wake potential," Planetary and Space Science, vol. 46, no. 5, pp. 541-543, 1998.

[14] P. K. Shukla, M. Y. Yu, and R. Bharuthram, "Linear and nonlinear dust drift waves," Journal of Geophysical Research: Space Physics, vol. 96, no. A12, pp. 21343-21346, 1991.

[15] A. A. Mamun, R. A. Cairns, and P. K. Shukla, "Solitary potentials in dusty plasmas," Physics of Plasmas, vol. 3, no. 2, pp. 702-704, 1996.

[16] A. A. Mamun, R. A. Cairns, and P. K. Shukla, "Effects of vortexlike and non-thermal ion distributions on non-linear dustacoustic waves," Physics of Plasmas, vol. 3, no. 7, pp. 2610-2614, 1996.

[17] A. A. Mamun, "Effects of ion temperature on electrostatic solitary structures in nonthermal plasmas," Physical Review E: Statistical Physics, Plasmas, Fluids, and Related Interdisciplinary Topics, vol. 55, no. 2, pp. 1852-1857, 1997.

[18] R. Z. Sagdeev, "Cooperative phenomena and shock waves in collisionless plasmas," Reviews of Plasma Physics, vol. 4, pp. 2391, 1966

[19] H. Washimi and T. Taniuti, "Propagation of ion-acoustic solitary waves of small amplitude," Physical Review Letters, vol. 17, no. 19 , pp. 996-998, 1966.
[20] A. A. Mamun and P. K. Shukla, "Solitary potentials in cometary dusty plasmas," Geophysical Research Letters, vol. 29, no. 18, p. 1870, 2002.

[21] C. Bedi, T. S. Gill, and A. S. Bains, "Four component magnetized dusty plasma containing non-thermal electrons," Journal of Physics: Conference Series, vol. 208, no. 1, Article ID 012037, 2010.

[22] S. Tasnim, A. Mannan, and A. A. Mamun, "Electrostatic solitary structures in a four-component adiabatic dusty plasma," Astrophysics and Space Science, vol. 337, no. 1, pp. 261-267, 2012.

[23] T. Akhter, M. M. Hossain, and A. A. Mamun, "Obliquely propagating solitary waves in a four-component magnetized dusty plasma," African Review of Physics, vol. 8, no. 0012, pp. 75-81, 2013.

[24] S. Sebastian, G. Sreekala, M. Michael et al., "Solitary waves in a five-component dusty plasma with kappa described electrons and ions," International Journal of Science and Research, vol. 3, no. 8, pp. 1301-1306, 2014.

[25] C. Tsallis, "Possible generalization of Boltzmann-Gibbs statistics," Journal of Statistical Physics, vol. 52, no. 1-2, pp. 479-487, 1988.

[26] A. M. El-Hanbaly, E. K. El-Shewy, M. Sallah, and H. F. Darweesh, "Nonlinear dust acoustic waves in dissipative space dusty plasmas with superthermal electrons and nonextensive ions," Communications in Theoretical Physics, vol. 65, no. 5, pp. 606-612, 2016

[27] M. Tribeche and P. K. Shukla, "Charging of a dust particle in a plasma with a non extensive electron distribution function," Physics of Plasmas, vol. 18, no. 10, p. 103702, 2011.

[28] R. Amour and M. Tribeche, "Variable charge dust acoustic solitary waves in a dusty plasma with a q-nonextensive electron velocity distribution," Physics of Plasmas, vol. 17, no. 6, 2010.

[29] S. A. El-Wakil, M. A. Zahran, E. K. El-Shewy, and H. G. Abdelwahed, "Solitary waves in space dusty plasma with dust of opposite polarity," in Proceedings of the 7th Conference on Nuclear and Particle Physics, pp. 323-333, Sharm El-Sheikh, Egypt, 11-15 Nov. 2009.

[30] K. Roy, P. Chatterjee, and S. Kundu, "Dust acoustic dressed solitons in a four component dusty plasma with nonthermal electron," Advances in Space Research, vol. 50, no. 9, pp. 12881293, 2012

[31] W. F. El-Taibany, "Nonlinear dust acoustic waves in inhomogeneous four-component dusty plasma with opposite charge polarity dust grains," Physics of Plasmas, vol. 20, no. 9, 2013.

[32] A. E. Dubinov, "On a widespread inaccuracy in defining the Mach number of solitons in a plasma," Plasma Physics Reports, vol. 35, no. 11, pp. 991-993, 2009.

[33] V. V. Prudskikh, "Ion-acoustic solitons in Bi-Ion dusty plasma," Plasma Physics Reports, vol. 34, no. 11, pp. 955-962, 2008.

[34] A. E. Dubinov and D. Y. Kolotkov, "Ion-acoustic super solitary waves in dusty multispecies plasmas," IEEE Transactions on Plasma Sciences, vol. 40, no. 5, pp. 1429-1433, 2012.

[35] A. E. Dubinov, D. Y. Kolotkov, and M. A. Sazonkin, "Supernonlinear waves in plasma," Plasma Physics Reports, vol. 38, no. 10, pp. 833-844, 2012. 


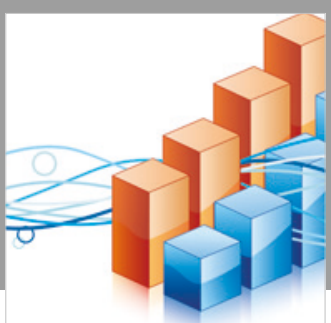

Advances in

Operations Research

\section{-n-m}
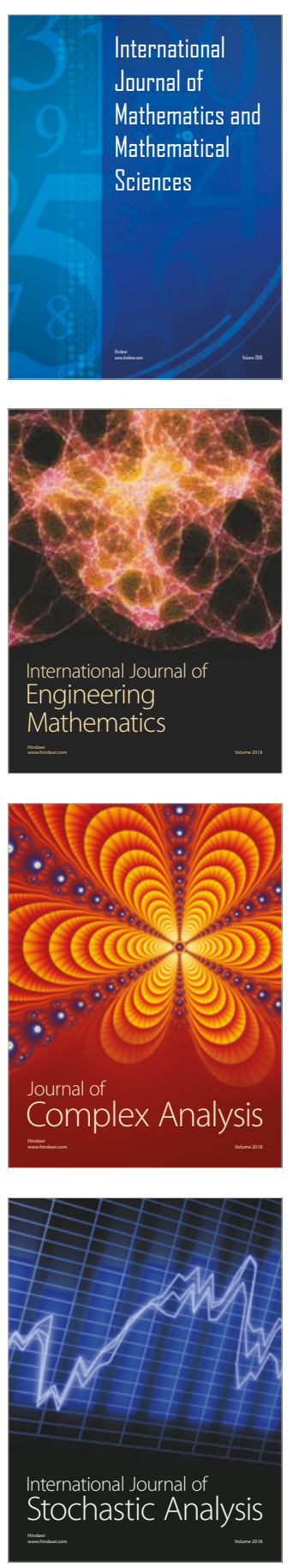
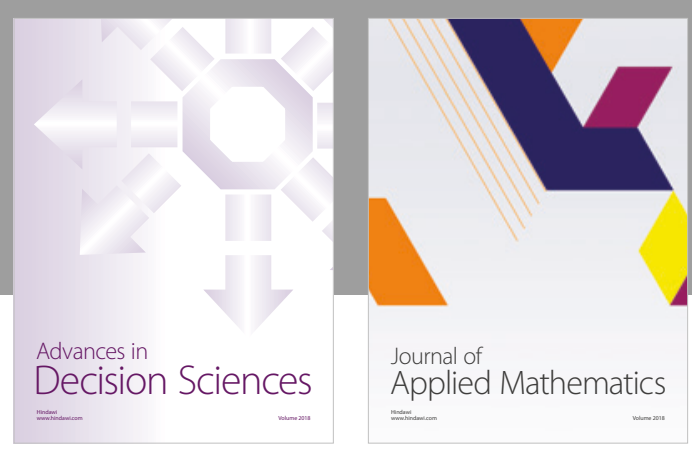

Journal of

Applied Mathematics
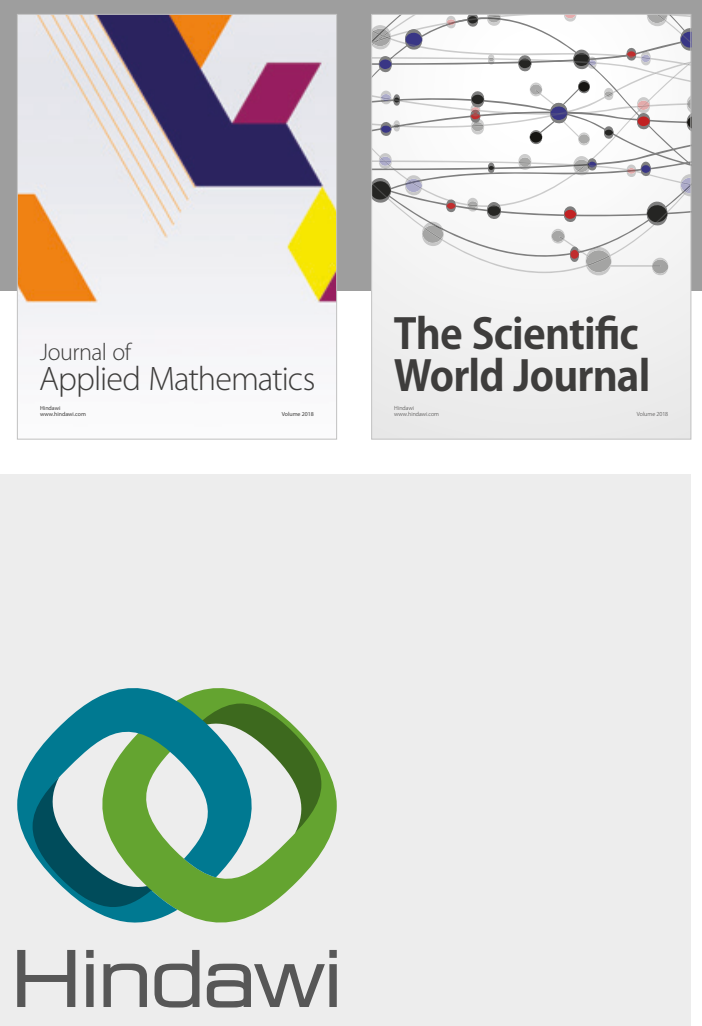

Submit your manuscripts at

www.hindawi.com

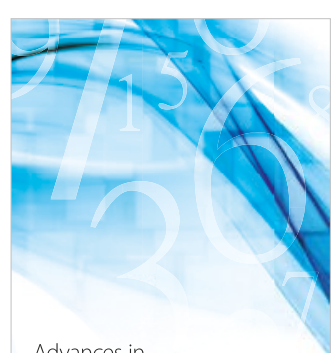

Advances in
Numerical Analysis
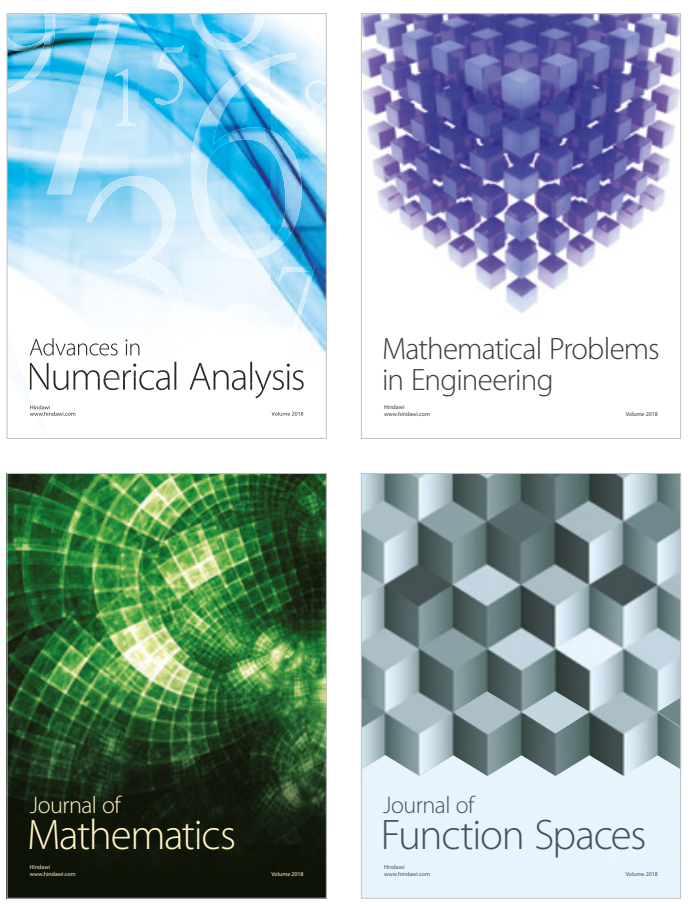

Mathematical Problems in Engineering

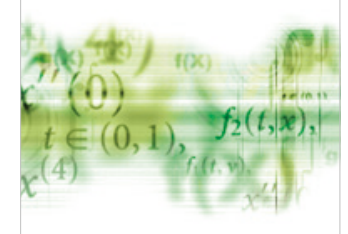

International Journal of

Differential Equations

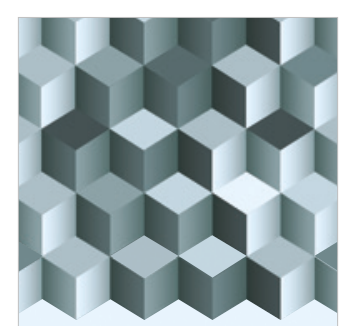

Journal of

Function Spaces

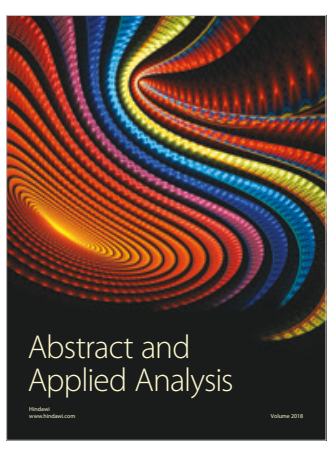

The Scientific

World Journal

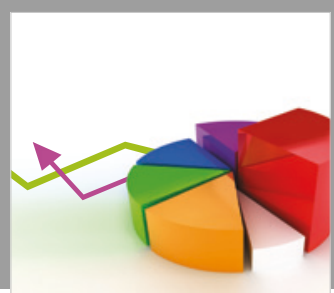

Journal of

Probability and Statistics
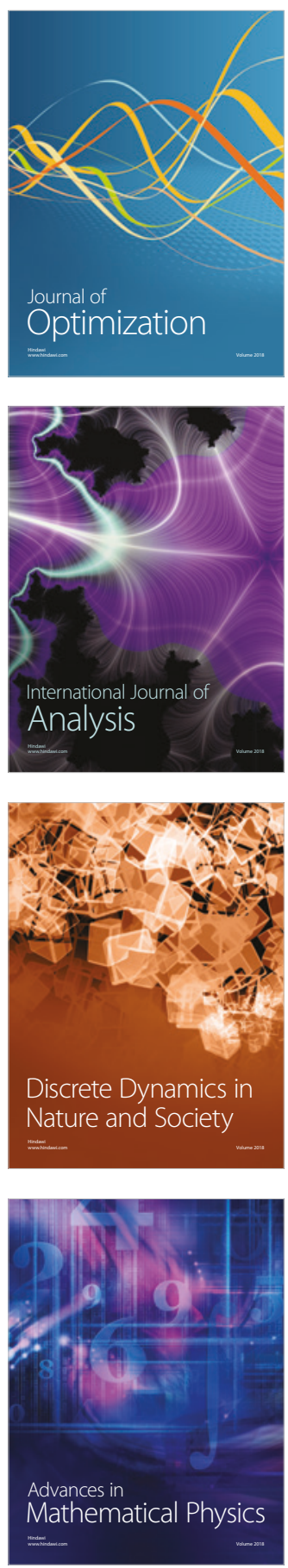